\title{
Modelo de Gestión de la Innovación para mejorar la efectividad en el lanzamiento de nuevos productos en Fundación Malecón 2000
}

\section{Innovation Management Model to improve effectiveness in the launch of new products in Malecon 2000 Foundation}

\author{
Ing. Wellington Armando Martillo Calderón ${ }^{1}$ \\ martillonet@yahoo.com \\ https://orcid.org/0000-0002-9055-1847 \\ PhD. Otto Suárez Rodríguez ${ }^{2}$ \\ docenteinvestigador@uteg.edu.ec \\ https://orcid.org/0000-0002-2515-7026
}

Recibido: 01/07/2020, Aceptado: 01/09/2020

\begin{abstract}
RESUMEN
En la actualidad, la administración de la innovación representa un aspecto muy importante en las organizaciones a nivel mundial, pero es necesario que se adopte un enfoque estratégico porque la innovación en sí misma no es suficiente para alcanzar el éxito. En este trabajo se estableció el objetivo de elaborar un modelo de gestión de la innovación aplicable a Fundación Malecón 2000 de la ciudad de Guayaquil, con un enfoque estratégico, para mejorar la efectividad en el lanzamiento de nuevos productos. Se aplicaron sendas encuestas a una muestra de visitantes del Malecón 2000 y a los empleados de la Fundación. Uno de los principales resultados obtenido de los visitantes fue la buena predisposición a aportar con ideas de nuevos productos y por parte de los empleados se determinó que es limitado el aporte de los colaboradores al no estar integrados en el proceso de innovación de la organización. La Fundación Malecón 2000 tiene la oportunidad de adoptar un modelo de gestión alineado al modelo Stage-Gate, que incorpore conceptos relevantes de la innovación como la cadena de valor, el clima organizacional, el Modelo de Innovación Abierta y la gestión de riesgos en la innovación, para lograr la satisfacción plena de los visitantes, así como el retorno satisfactorio de la inversión.
\end{abstract}

Palabras clave: gestión, modelos de innovación, efectividad, lanzamiento de productos, Fundación Malecón 2000

\footnotetext{
ABSTRACT

${ }^{1}$ Especialista en Sistemas Fundación Malecón 2000, Guayaquil, Ecuador

2 Universidad Tecnológica Empresarial de Guayaquil, Ecuador
}

Currently, innovation management represents a very important aspect in organizations worldwide, but it's necessary to take a strategic approach because innovation alone is not enough to achieve success. Therefore, the objective of this 


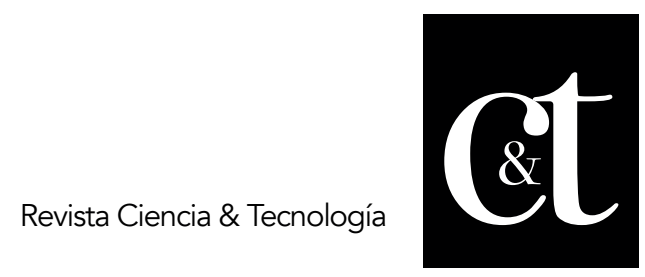

No. 28, 31 de octubre de 2020

ISSN impreso: 1390 - 6321

ISSN online: 2661 - 6734

study was to propose an innovation management model applied to the Malecon 2000 Foundation of the city of Guayaquil, with a strategic focus, to improve the effectiveness of the launching of new products. Two surveys were administered, one to a sample of visitors of the Malecon 2000 and the other one to the employees of the Foundation. One of the key results obtained from the visitors was their willingness to contribute with ideas of new products, and from the employees, it was determined that their contribution was limited because they are not fully integrated within the innovation process of the organization. The Malecon 2000 Foundation has the opportunity to adopt a model based on the Stage-Gate Model of Innovation, which seeks to incorporate relevant innovation concepts such as chain value, organizational climate, Open Innovation Model, and innovation risk management, in order to achieve full satisfaction of the visitors as well as a satisfactory investment return.

Keywords: management, innovation models, effectiveness, product launching, Malecon 2000 Foundation

\section{Introducción}

La innovación es uno de los temas más importantes y complejos a los que las organizaciones se enfrentan en la actualidad, ya que es clave para el éxito de las mismas. La innovación consiste en la aplicación de ideas, ya sea que se utilicen para desarrollar o mejorar productos, procesos, servicios, sistema de marketing o de gestión (Tohidi \& Jabbari, 2011).

Si se considera el Índice de Innovación Global (Cornell, INSEAD, \& WIPO, 2018), Ecuador se encuentra en la posición 93 de 127 países, por lo tanto, es imperativo que las instituciones ecuatorianas se enfoquen en impulsar más la creación y difusión del conocimiento, el diseño de nuevos productos y servicios, mejorando la infraestructura en general, y vinculando la universidad, organizaciones y la investigación.

Las organizaciones sin fines de lucro también pueden ser competitivas, haciendo uso de la gestión de estrategias de innovación, lanzando nuevos y mejores productos y aplicando estrategias para mitigar el riesgo, que les permita ser sostenibles para cumplir con sus metas de manera eficiente y eficaz; en beneficio de los integrantes de la sociedad para la cual realizan sus actividades.

La Fundación Malecón 2000 de la ciudad de Guayaquil es una organización privada, sin fines lucro, encargada de la administración del Malecón 2000 y del Estero Salado, con un presupuesto anual de US\$ 12 millones aproximadamente (Deloitte, 2015), cuenta actualmente con varios productos, entre los cuales destacan los siguientes: parqueos, áreas comerciales; sitios de entretenimiento familiar como cines y parques. Por su trayectoria, la Fundación es pionera en materia de proyectos de regeneración urbana en el Ecuador.

La Fundación tiene como misión "Preservar el Malecón Simón Bolívar y Malecón del Estero Salado, como íconos turísticos y de entretenimiento" y su visión es: "Ser la primera opción turística y de entretenimiento en Guayaquil y Latinoamérica".

Desde su creación en el año 1997, la Fundación ha sido una institución innovadora, con exitosos proyectos de regeneración urbana, sin embargo, no ha utilizado un 


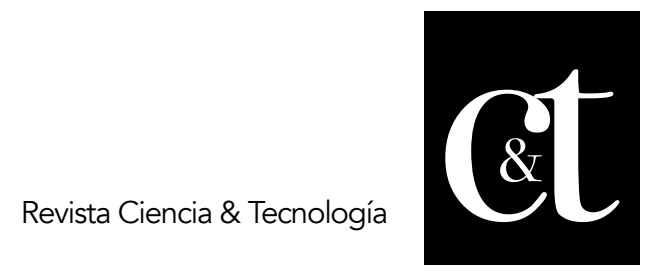

No. 28, 31 de octubre de 2020

ISSN impreso: 1390 - 6321

ISSN online: 2661 - 6734

modelo estandarizado de estrategia de innovación para el desarrollo y lanzamiento de nuevos productos, por lo que en ocasiones no se han alcanzado los resultados esperados.

La institución fue concebida para regenerar el Malecón Simón Bolívar, y encargarse de su administración y mantenimiento, cabe indicar que la Fundación no recibe financiamiento del sector público, por lo que es responsable de generar por si misma los ingresos económicos para los gastos de administración y mantenimiento de las instalaciones a su cargo.

Por lo cual, el objetivo de la presente investigación es elaborar un modelo para gestionar la innovación de productos para que la Fundación logre un mayor grado de éxito, mejorando todo el proceso de innovación, desde las ideas, hasta el lanzamiento de nuevos productos, y poder conseguir una mayor efectividad en las estrategias de innovación.

\section{Desarrollo}

Una serie de factores, entre ellos la globalización, los avances en la tecnología de la información y las comunicaciones (TIC) y la creciente complejidad tecnológica, han inducido a las empresas a participar cada vez más en colaboraciones de innovación, y pueden adoptar innovaciones abiertas para administrar y reducir los costos y riesgos asociados con el desarrollo de nuevos productos, y lograr mantenerse por delante de sus competidores (Brant \& Lohse, 2014).

Así también Igartua (2009) señala que, aunque existe un extenso número de estudios sobre innovación en las empresas, la evidencia sobre el papel de herramientas de gestión de la innovación es escasa, esto significa que aún falta mucho por llegar a las organizaciones con modelos de gestión de la innovación que aporten de forma concreta.

En la misma línea, la aplicación de modelos innovadores en organizaciones no gubernamentales (ONG) o sin fines de lucro, no está lo suficientemente documentada, en parte por las dificultades inherentes que enfrentan estas organizaciones para la adopción de propuestas innovadoras dentro de sus modelos de gestión (Ramos et al., 2018).

En ese orden de ideas, la concepción e implantación de cambios significativos en el producto, el proceso, el marketing o la organización de la empresa con el propósito de mejorar los resultados es lo que se entiende por innovación. Los cambios que resultan innovadores se logran mediante la aplicación de nuevos conocimientos y tecnología que pueden ser internos de las organizaciones, por medio de colaboración externa, a través de servicios de asesoramiento, o también por la compra de nuevas tecnologías (Manual de Oslo, 2005).

\section{Modelos de Innovación}

Aunque el papel de la innovación ha sido abordado en la literatura desde el ámbito de los negocios, ésta también puede ser aplicada en organizaciones sin fines de lucro, para el efecto existe una gama de modelos de innovación; se debe tener en cuenta que un modelo es un conjunto de variables y sus relaciones; en otras palabras, un modelo es una representación que abstrae algunos factores explicativos esenciales de un fenómeno y representa sus relaciones. En resumen, un modelo es una representación de la realidad en una forma simplificada (Godin, 2015). 


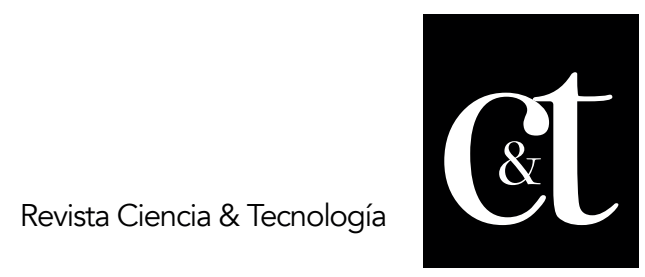

No. 28, 31 de octubre de 2020

ISSN impreso: 1390 - 6321

ISSN online: 2661 - 6734

\section{Modelo de Innovación Abierta}

Desde que Henry Chesbrough (2003) presentó el concepto de innovación abierta, este modelo se ha convertido en un nuevo paradigma para administrar la innovación en las organizaciones. La innovación abierta impulsa a las empresas para que usen ideas internas y externas. La innovación abierta es el uso de flujos internos y externos de conocimiento para acelerar la innovación y ampliar los mercados para el uso de dicha innovación (Brant \& Lohse, 2014).

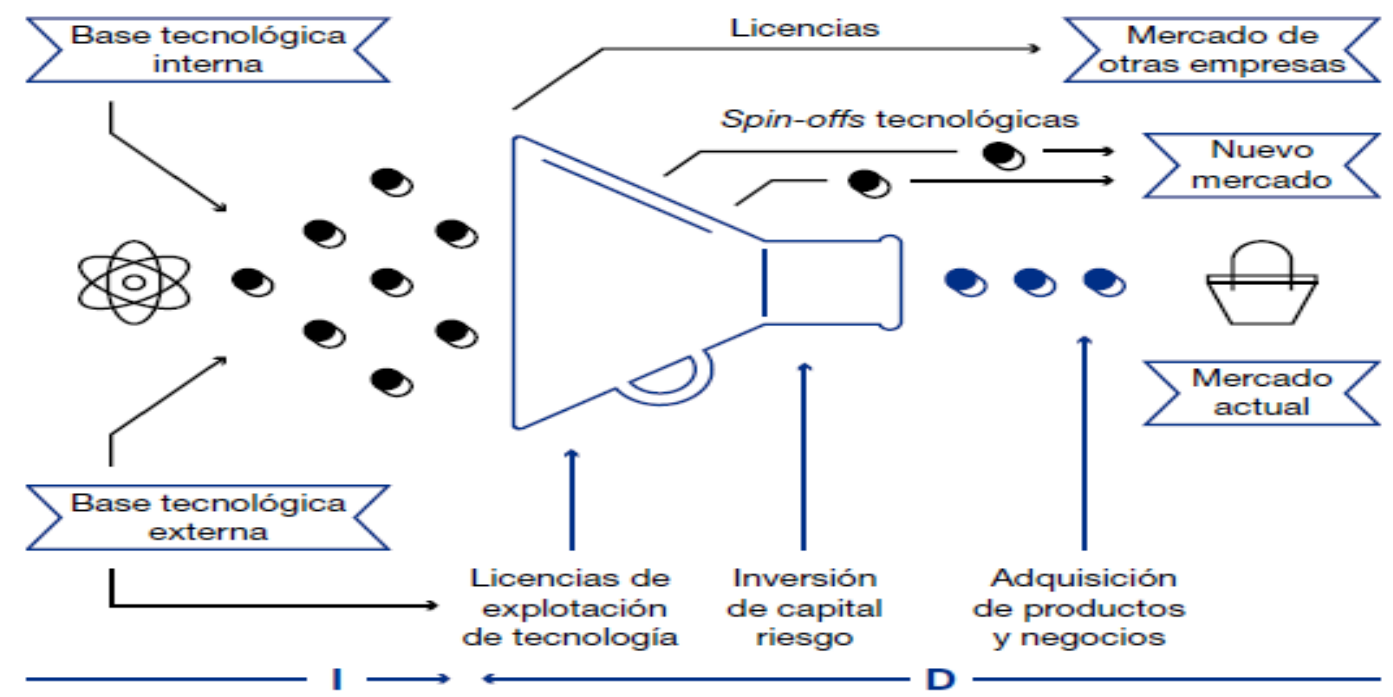

Figura 1. Modelo de Innovación Abierta

Fuente: Chesbrough (2014)

La innovación abierta se podría interpretar como la antítesis del modelo tradicional de integración vertical, en el que las actividades internas de innovación conducen a productos y servicios desarrollados dentro de la empresa, que a continuación los distribuye. La innovación abierta es el uso de flujos internos y externos de conocimiento para acelerar la innovación interna y ampliar los mercados para el uso externo de dicha innovación (Chesbrough, 2014).

Los procesos de innovación abierta (ver Fig. 1), combinan ideas internas y externas en plataformas, arquitecturas y sistemas; utilizan modelos de negocio para definir los requisitos para estas arquitecturas y sistemas. Estos modelos de negocio acceden a ideas tanto externas como internas para crear valor al tiempo que definen mecanismos internos para reclamar una parte de ese valor.

\section{Modelo Stage-Gate}

El modelo Stage-Gate es reconocido y ampliamente aceptado en compañías de todo el mundo debido a que permite poner orden en el proceso, muchas veces caótico, de la innovación de productos. Robert Cooper, lo describe como un modelo para mover un nuevo producto desde una idea hasta el lanzamiento utilizando varias etapas y puertas que se activan según los criterios de decisión más convenientes para los intereses de la empresa (Edgett,2018). 


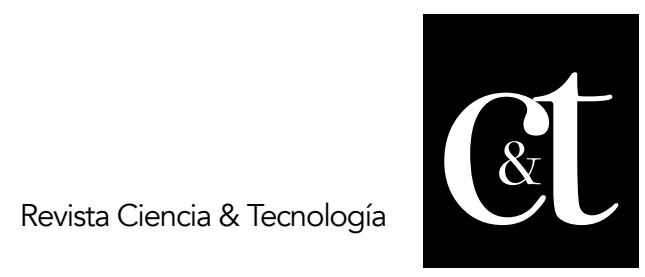

No. 28, 31 de octubre de 2020

ISSN impreso: 1390 - 6321

ISSN online: 2661 - 6734

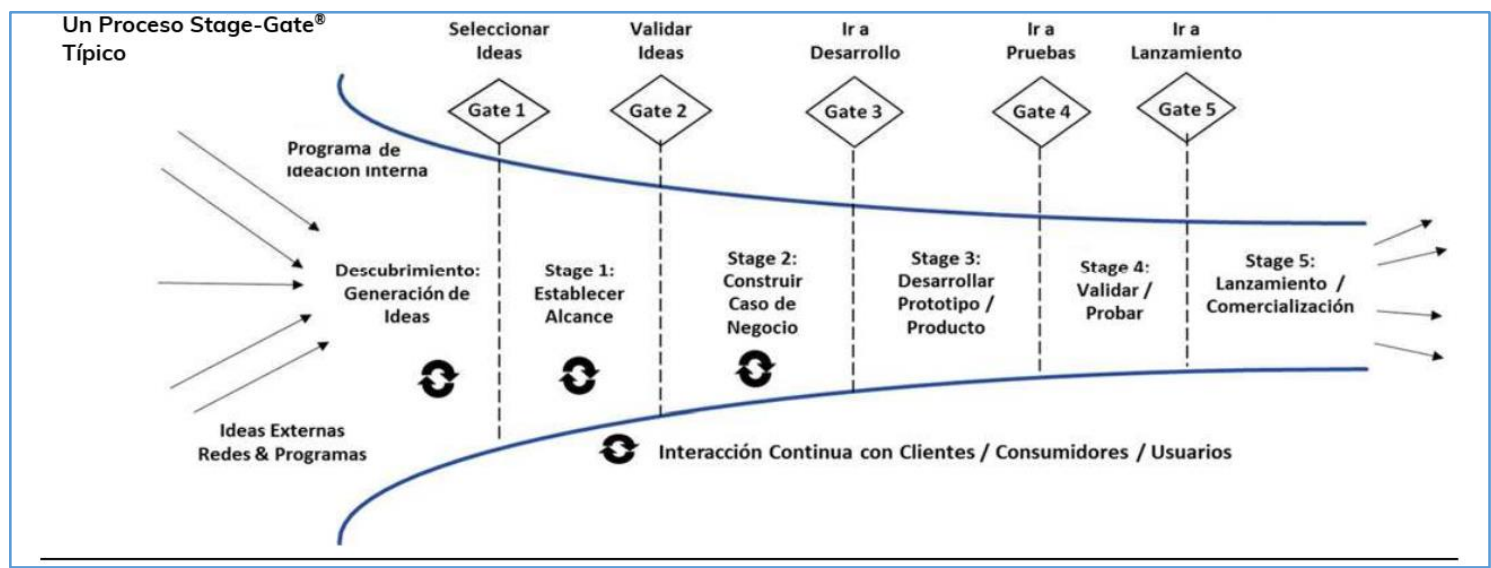

Figura 2. Modelo Stage-Gate

Fuente: Edgett (2018)

Entre las organizaciones de clase mundial que utilizan Stage-Gate se encuentran 3M, PepsiCo, Kellogs, Bayer, Lego y la Oficina Británica de Comercio. Stage-Gate divide el proceso de innovación en un conjunto predeterminado de etapas. Cada etapa incluye un conjunto de actividades, factores críticos de éxito y toma de decisiones para poder pasar a la siguiente etapa del proceso.

El modelo Stage-Gate es una hoja de ruta conceptual y operativa para mover un nuevo proyecto de producto desde la idea hasta el lanzamiento, un plan para administrar el proceso de innovación del producto para mejorar la eficacia y la eficiencia. Y se basa en las mejores prácticas y en los factores críticos para el éxito, de modo que cuando se sigue adecuadamente, el éxito está casi asegurado (Edgett, 2018).

Modelo de Efectividad de la Innovación de Klein \& Sorra

La efectividad de la innovación surge como un conjunto de aspectos que inician con un clima organizacional, valores, compromiso y habilidades a favor de la innovación. (Klein \& Knight, 2005). Los valores de innovación, pueden variar entre individuos, entre grupos o entre organizaciones; por lo tanto, debe enfocarse en las diferencias entre las organizaciones y las diferencias entre los grupos en cuanto a los valores de innovación, porque se ajusta principalmente como valores compartidos de los usuarios de una organización como un todo o grupos diferentes de usuarios específicos.

\section{Modelo de Madurez de la Innovación}

Modelo desarrollado por Carnegie Mellon University; incluye tres factores que son esenciales para un programa de innovación eficaz: personas, procesos y herramientas, incluye todo el ciclo de vida completo del producto desde la idea hasta el lanzamiento y hasta el final de la vida útil (Nauyalis, 2018). Este modelo brinda a las organizaciones la posibilidad de clasificarse según la solidez de su programa de innovación, donde el nivel 1 representa el nivel más bajo de madurez, mientras que el nivel 5 es el más alto, donde la innovación está altamente optimizada. 


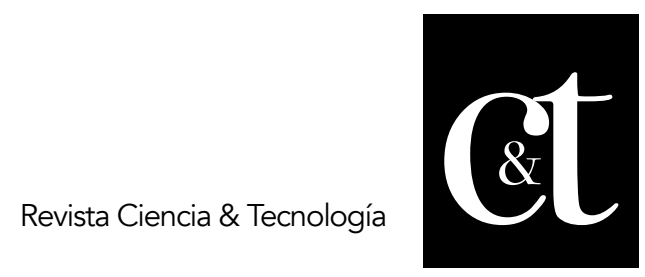

No. 28,31 de octubre de 2020

ISSN impreso: 1390 - 6321

ISSN online: 2661 - 6734

\section{Administración del Riesgo en la Innovación}

Administrar riesgos en la innovación es un aspecto importante, porque está presente en el desarrollo de nuevos productos; y además produce un efecto positivo e impacto significativo en el rendimiento del nuevo producto de una empresa (Ahmed, 2017).

Es muy importante transferir, prevenir y reducir el riesgo; por lo que se debe analizar los factores de riesgo, transferir y controlar el riesgo, mejorar para resistir la capacidad del riesgo y mejorar constantemente la capacidad técnica para hacer frente a la crisis. Si se tiene un sistema eficaz de gestión de riesgos, la innovación tecnológica puede ser una implementación exitosa, la aversión al riesgo o negativa no es una solución efectiva.

Indicadores de Desempeño en Innovación (KPI)

Entre los indicadores que debe usar una organización para medir su desempeño en innovación se encuentran ROI, ventas, crecimiento de ventas, Payback, Flujo de Caja, satisfacción del cliente, retención del cliente, y desarrollo del empleado (Sawang, 2011). Una organización innovará productivamente aplicando algunos de los siguientes Factores Críticos de Éxito (Govindarajan, 2011): caso convincente para la innovación, visión inspiradora y compartida del futuro, una agenda de innovación estratégica totalmente alineada y participación visible de la alta gerencia.

\section{Software de Gestión de la Innovación}

El proceso de gestión de la innovación requiere el uso de ciertas herramientas de gestión que ayudan a los gerentes y otras entidades a una plataforma común y les permiten avanzar hacia un objetivo común. Estas herramientas de gestión de la innovación pueden ser desde una simple sesión de lluvia de ideas hasta algo más complejo, como la planificación y la creación de prototipos.

La empresa global de consultoría empresarial Forrester, referida por Visitacion (2018), afirma que las soluciones de TI más importantes para administrar la innovación en las organizaciones son Spigit PlanView, Hype Innovation, KPMG Innovation, entre otras.

\section{Estrategia de Innovación}

La estrategia de innovación es la decisión de una organización que determina la medida y forma de utilizar la innovación para ejecutar su estrategia comercial con el intento de alcanzar ciertos niveles de desempeño (Aydinoglu, 2007). En ese sentido, agrega Turriago (2014), las organizaciones tienen las siguientes estrategias de innovación entre otras opciones:

La estrategia ofensiva de innovación es aquella que pretende conseguir el liderazgo del mercado, ubicándose en la cabeza de sus competidores en la introducción de nuevos productos, para lo cual la empresa deberá ser muy intensiva en investigación y en la defensa de patentes. Tendrá una visión a largo plazo, lo que la llevará a asumir altos riesgos.

Por su parte, la estrategia defensiva de innovación está muy vinculada a lograr una diferenciación del producto. La empresa no quiere ser la primera, pero tampoco quiere ser dejada atrás en los procesos de cambio tecnológico. Se caracteriza porque la organización adopta actitudes de reacción y adaptación a los cambios de sus competidores y deberá dedicar recursos a la educación y capacitación, tanto de sus clientes como de sus colaboradores. 


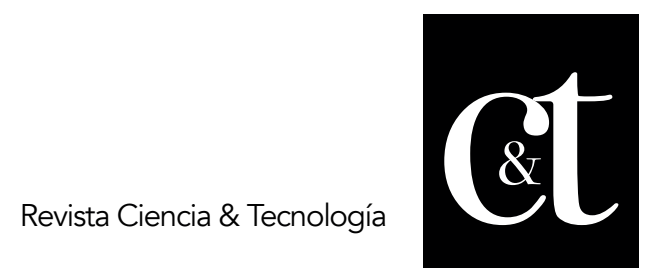

No. 28, 31 de octubre de 2020

ISSN impreso: 1390 - 6321

ISSN online: 2661 - 6734

\section{Metodología}

Para la presente investigación se utilizó el método analítico-sintético, pues se explica con base a los resultados específicos para tener una visión total de problema, sintetizando y uniendo hechos aislados. El método empírico utilizado fue la encuesta, por medio de esta técnica se obtienen datos estandarizados de la población a través de interrogantes cuantitativas y cualitativas.

La unidad de análisis es Fundación Malecón 2000, la población está constituida, por una parte, por los visitantes del Malecón 2000 y, por otro lado, los colaboradores de diferentes áreas administrativas de la Fundación; de cada una de estas poblaciones se seleccionaron muestras, a cuyos individuos se solicitó consentimiento informado.

En el caso de las personas que visitan el Malecón 2000, según información del portal web Guayaquil es Mi Destino, la ciudad recibió la visita de 2'111.260 turistas en el año 2017. De los cuales 1'418.556 son nacionales y 692.704 visitantes extranjeros. De esta población de estudio, se obtuvo una muestra de 385 individuos para aplicar la encuesta, en los siguientes lugares: Parque Las Iguanas (Seminario), centro de Guayaquil y alrededores del Malecón 2000.

En cuanto a la población y muestra para encuestar a los colaboradores administrativos de la Fundación Malecón 2000, se consideró la población de 30 empleados administrativos con que cuenta la Fundación, siendo esta misma la muestra por tratarse de un número bajo de personas.

\section{Enfoque y diseño de la investigación}

La presente investigación utilizó el enfoque cuantitativo y el diseño aplicado es no experimental, esto debido que lo que se hace es observar un fenómeno tal como se da en su contexto natural, para posteriormente analizarlos (Hernández Sampieri, Fernández y Baptista, 2010).

Así también el alcance es descriptivo para detallar el escenario actual del proceso de innovación en la organización, para que con base a estadísticas y el análisis de los casos de innovación realizados en la Fundación se determine los modelos de innovación aplicables y recomendar un nuevo modelo de innovación para que la organización logre mayor efectividad.

La investigación tuvo también un componente exploratorio para esclarecer y delimitar los problemas empresariales poco estudiados (Vara-Horna, 2010), en efecto el presente trabajo de investigación buscó conocer, definir y esquematizar en detalle las etapas del proceso de innovación mediante una investigación de tipo bibliográfico y el estudio de algunas tesis sobre esta temática.

\section{Resultados y Discusión}

En la Fundación, actualmente no existe una metodología formal de gestión de la innovación. En lo que se refiere al proceso de innovación, se realizan reuniones semanales, para analizar nuevas opciones de productos, en las que participan colaboradores de diferentes áreas tales como marketing, comercial, administrativa y financiera, basándose en una lluvia de ideas en las que se analiza la factibilidad de llevar adelante la idea de un nuevo producto.

Según los resultados obtenidos en la encuesta aplicada a los visitantes del Malecón 2000 , se logró detectar que la mayoría, es decir, el 70\% estaría dispuesto a colaborar 
con ideas para mejorar los productos que lanza la Fundación, siempre y cuando existan los medios (ver Figura 3). En ese sentido, el 33.4\% de visitantes afirmó que les gustaría utilizar una App móvil para sugerencias, seguido de un $28.6 \%$ que preferiría un portal web.

\section{¿Le gustaría participar con ideas de nuevos productos en Malecón 2000?}

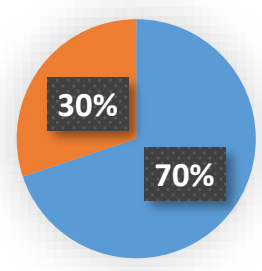

Figura 3. Disposición a colaborar con ideas de nuevos productos para el Malecón 2000

Fuente: Encuesta a visitantes

Acerca de los productos que los visitantes del Malecón prefieren utilizar manifestaron lo siguiente: el $35.8 \%$ de encuestados manifiesta que el producto que más utiliza es el parqueo del Malecón, posiblemente por su ubicación; luego un $31 \%$ de consultados utiliza las áreas comerciales, esto incluye el Centro Comercial, tiendas y locales a lo largo del Malecón. Así también un $16.8 \%$ de visitantes afirma que el producto que más utiliza son los parques de entretenimiento (Safaris); el $6.6 \%$ opta por el Cinema y un $2.1 \%$ utilizan el Teatro (ver Figura 4 ).

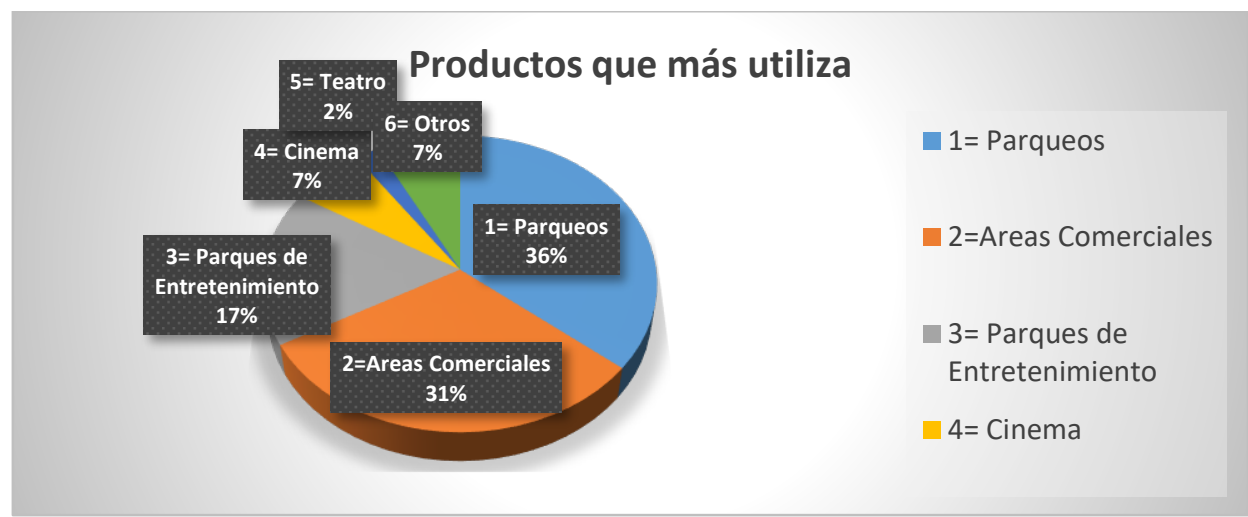

Figura 4. Productos que más utiliza

Fuente: Encuesta a visitantes

En cuanto al nivel de satisfacción de los visitantes (Ver Figura 5), se encontró que el $44 \%$ de los encuestados indican que están satisfechos con los productos del Malecón; el $13.3 \%$ se muestra muy satisfecho y el $1.8 \%$ está extremadamente satisfecho, es decir un $59 \%$ de visitantes tiene un buen nivel de satisfacción con los productos que ofrece el Malecón, y el $23.8 \%$ se sienten poco satisfechos y el $17.2 \%$ nada satisfechos, por lo que es importante definir las mejoras que ayuden a comprender al consumidor y desarrollar productos que sirvan como atractivos para incrementar el nivel de satisfacción. 


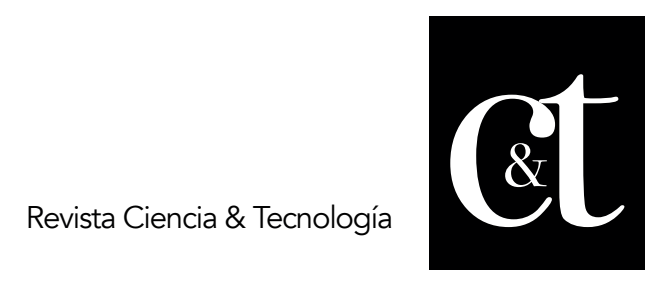

No. 28,31 de octubre de 2020

ISSN impreso: 1390 - 6321

ISSN online: 2661 - 6734

\section{Nivel de Satisfacción con los Productos del Malecón}

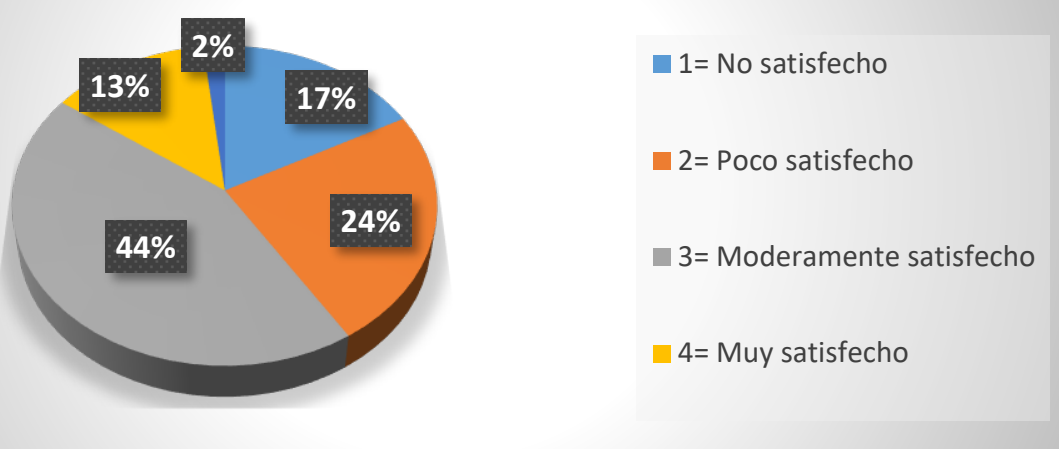

Figura 5. Nivel de Satisfacción con Productos

Fuente: Encuesta a visitantes

En cuanto a los resultados de la encuesta aplicada a los empleados administrativos de la Fundación, el $30 \%$ de encuestados afirma que participa en la creación de nuevos productos en la Fundación, esto significa que la organización está dejando de captar un $70 \%$ de ideas que podrían convertirse en nuevos productos, por lo que se deben adoptar las medidas necesarias para poder aprovechar el aporte de los colaboradores que no están integrados en el proceso de innovación de la organización.

La Fundación tiene la oportunidad de implementar Innova3000, un modelo de innovación de productos que puede ser utilizado en toda organización y que está centrado principalmente en el modelo Stage-Gate. La implementación de un modelo especializado como Innova 3000, según la encuesta, tendría un apoyo del $86.7 \%$ de los empleados (ver Tabla 1).

\section{Tabla 1. Conveniencia de utilizar un modelo especializado aplicable a la Fundación en futuros procesos de innovación}

\begin{tabular}{|c|c|c|c|c|c|}
\hline & & Frecuencia & Porcentaje & $\begin{array}{l}\text { Porcentaje } \\
\text { válido }\end{array}$ & $\begin{array}{l}\text { Porcentaje } \\
\text { acumulado }\end{array}$ \\
\hline \multirow[t]{3}{*}{ Válido } & Sí & 26 & 86.7 & 86.7 & 86.7 \\
\hline & NO & 4 & 13.3 & 13.3 & 100.0 \\
\hline & Total & 30 & 100.0 & 100.0 & \\
\hline
\end{tabular}

Fuente: Encuesta a empleados

\section{Propuesta}

El Modelo Innova3000 (ver Figura 6) incorpora actividades iniciales de pre-desarrollo (justificación de negocio soportada por los clientes y factibilidades preliminares), actividades de desarrollo a nivel de marketing y operaciones, y actividades lanzamiento al mercado en un proceso de negocio completo. 


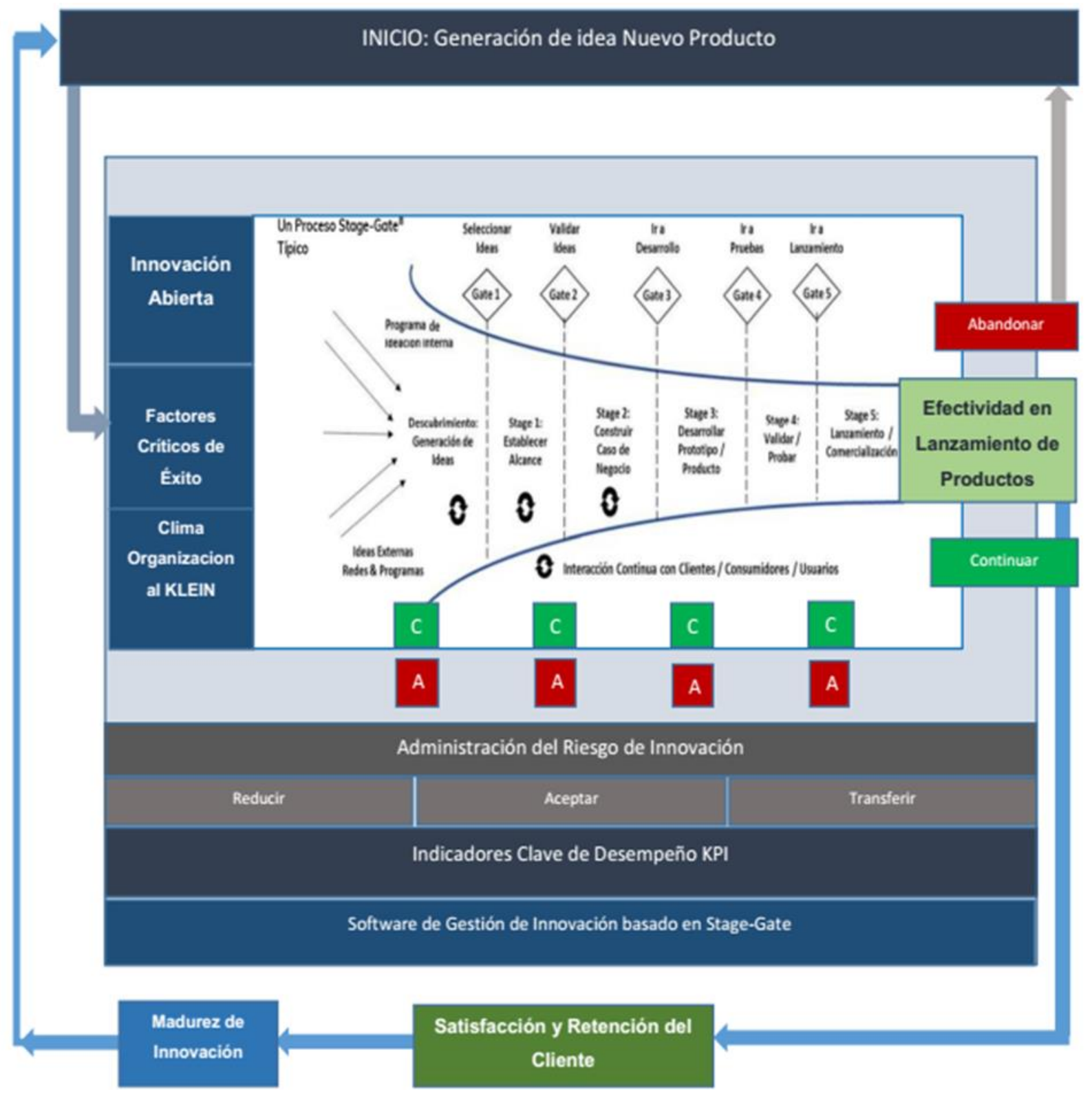

Figura 6. Modelo INNOVA3000

Fuente: Elaboración propia

La Fundación invertiría alrededor de un año en adoptar el modelo, entre su introducción y socialización, la capacitación, la selección del software de apoyo, la implementación y puesta en marcha. El costo de implementar el modelo INNOVA3000 apoyándose en un software es de US\$ 24 mil dólares, y se calcula que el ROI es del $62 \%$ con un Payback de 1.2 años.

El Modelo Innova3000, centrado en el modelo Stage-Gate, incorpora conceptos relevantes de la innovación como: Cadena de Valor de la Innovación, Modelo de Klein \& Sorra (clima organizacional), Modelo de Innovación Abierta y la gestión de riesgos en la innovación, para lograr llegar a la satisfacción del cliente. 


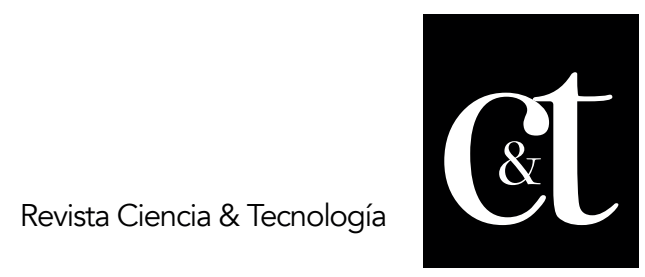

No. 28, 31 de octubre de 2020

ISSN impreso: 1390 - 6321

ISSN online: 2661 - 6734

Funcionamiento del Modelo INNOVA3000

En el Modelo INNOVA3000 las actividades están diseñadas para reunir información y disminuir progresivamente la incertidumbre. Los resultados de este análisis integrado se convierten en un conjunto de información que proporciona la entrada a las reuniones de toma de decisión (gates). Anterior a cada etapa, un proyecto pasa a través de una compuerta en la que se toma la decisión continuar o no invirtiendo en el proyecto (una decisión de avanzar/parar).

Esto sirve como punto de control de control de calidad con tres objetivos: evaluar la justificación del negocio, asegurar la calidad de ejecución y aprobar el plan de proyecto y recursos. Cada compuerta tiene un propósito distinto. Por ejemplo, la primera compuerta es un filtro suave de nuevas ideas, mientras que la segunda compuerta es más rígida, donde se debe justificar la decisión de la compuerta que aprueba al proyecto para entrar a la etapa de desarrollo que es mucho más costosa.

Cada proyecto se mide contra un conjunto claramente definido de criterios de éxito. Los criterios deben ser robustos para ayudar a identificar los productos que serán exitosos más prontamente. El proceso incluye los siguientes criterios:

- Ajuste Estratégico, es el grado de alineación con el negocio y estrategia de innovación.

- Producto y Ventaja Competitiva, es el grado en el que el producto potencial ofrece mayores beneficios al cliente y su impacto en la ventaja competitiva.

- Atractivo del Mercado, es el tamaño del mercado.

- Sinergias/Competencias centrales, apalancar las competencias centrales en mercadeo, ventas, operaciones, y distribución.

- Viabilidad técnica, es el grado de complejidad técnica y el tamaño de la brecha técnica.

- Recompensa Financiera/Riesgos, Payback y el riesgo financiero.

\section{Conclusiones}

Se ha constatado que actualmente, la Fundación Malecón 2000 no cuenta con un modelo de gestión de la innovación. El proceso de innovación de productos se realiza en reuniones en las que se utiliza básicamente la lluvia de ideas, y en cuanto a la tecnología se utiliza una suite de ofimática.

Según las encuestas realizadas a los visitantes de Malecón 2000, estos se muestran a favor de contar con un medio que les permita participar con ideas nuevas. Mientras tanto, la mayoría de colaboradores administrativos están interesados en que se les facilite un modelo que sirva como guía para mejorar la innovación y por ende aumentar el éxito en los nuevos productos.

Para la Fundación, no es suficiente basarse en un único modelo de gestión de la innovación, por lo que se ha determinado que modelos enfocados en la innovación por fases, en innovación abierta, la madurez de la organización para innovar, la cadena de valor de la innovación, pueden entre todos aportar los componentes necesarios para conformar un modelo más aplicable en la Fundación.

Los factores críticos de éxito más importantes para la Fundación son en primer lugar poder escuchar a los consumidores (visitantes) y a los colaboradores administrativos, segundo utilizar un modelo que tenga un componente de tecnología de información 


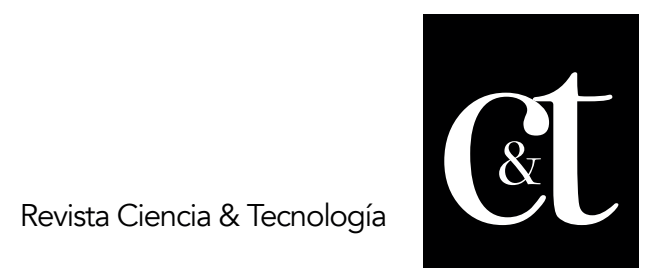

No. 28, 31 de octubre de 2020

ISSN impreso: 1390 - 6321

ISSN online: 2661 - 6734

para automatizar el proceso de innovación de nuevos productos y tercero poder contar con el respaldo de la gerencia para poder implementar todas las mejoras.

Un sistema informático especializado en la gestión de la innovación puede ser considerado a partir del ranking de soluciones creado por empresas de consultoría como Forrester, entre los cuales se encuentran PlanView, SAP Innovation Management, Sopheon, entre otros, dependiendo del costo que la Fundación esté dispuesta a invertir.

Finalmente, el modelo INNOVA3000 está enfocado de manera estratégica, de tal forma que puede servir para mejorar los resultados que necesita la Fundación en el desarrollo y lanzamiento de nuevos productos, aprovechando las ideas externas e internas y las ventajas competitivas que tiene la organización.

\section{Referencias bibliográficas}

Ahmed, R. (2017). Risk Mitigation Strategies in Innovative Projects. En Key Issues for Management of Innovative Projects. INTECH. Obtenido de: http://www.intechopen.com/books/key-issues-for-managementof-innovativeprojects

Aydinoglu, B. (2007). Innovation strategy measurement: Development of an assessment tool to measure 'Innovation Strategy' fitness of companies. Tesis de Maestría, TU Delft.

Brant, J., \& Lohse, S. (2014). The Open Innovation Model. ICC (International Chamber of Commerce) Innovation and Intellectual Property, Research Paper No. 2

Chesbrough, H. (2014). Innovación abierta. Innovar con éxito en el siglo XXI. Reinventar la empresa en la era digital OpenMind BBVA. Obtenido de: https://www.bbvaopenmind.com/articulos/articuloinnovacion-abierta-innovarcon-exito-en-el-siglo-xxi/

Cornell, INSEAD, \& WIPO (2018). Global Innovation Index 2018. https://www.globalinnovationindex.org/gii-2018-report

Deloitte (2015). Informe de Auditoría 2015. Obtenido de: http://malecon.org.ec/downloads/ley-detransparencia/antigua/presupuestos/g/informe_de_auditoria_deloitte_2015.pdf

Edgett, S. (2018). The Stage-Gate Model: An overview. Stage-Gate International. Obtenido de: content/uploads/2018/06/wp10english.pdf https://www.stage-gate.com/wp-

Godin, B. (2015). Models of innovation: Why models of innovation are models, or what work is being done in calling them models? Social Studies of Science, 45, 570-596.

Govindarajan, V. (2011). Innovation's Nine Critical Success Factors. Harvard Business Review. Obtenido de: https://hbr.org/2011/07/innovations-9-criticalsuccess

Hernández Sampieri, R., Fernández, C. y Baptista, M. (2010). Metodología de la Investigación. 5ta. Edición. México: McGraw Hill. 


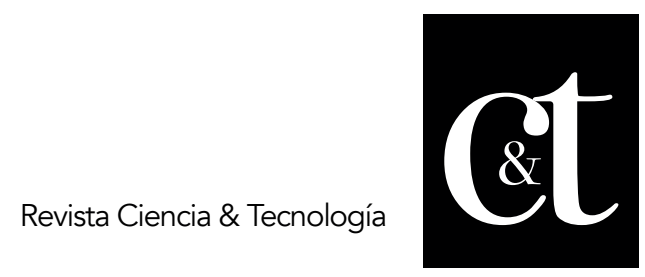

No. 28, 31 de octubre de 2020

ISSN impreso: 1390 - 6321

ISSN online: 2661 - 6734

Igartua, J. (2009). Gestión de la innovación en la empresa vasca. Tesis doctoral. Universitat Politécnica de Valencia.

Klein, K. J., \& Knight, A. P. (2005). Innovation implementation: Overcoming the challenge. Current Directions in Psychological Science, 14(5), 243-246.

Nauyalis, C. (2018). A New Framework for Assessing Your Innovation Program: Introducing The Innovation Management Maturity Model. Plainview, Inc.

OCDE Eurostat (2005). Manual de Oslo - Guía para la Recogida e Interpretación de Datos sobre Innovación.

Ramos, J., Luque, O., Martínez-Tur, V., Patras, L., Estreder, Y. \& Peñarroja, V. (2018). Oportunidades y barreras a la innovación: un estudio cualitativo en organizaciones del tercer sector. Instituto para la calidad de las ONG, Madrid.

Sawang, S. (2011). Key Performance Indicators for Innovation Implementation: Perception vs. Actual Usage Asia Pacific Management Review 16(1) (2011) 2329.

Tohidi, H. \& Jabbari, M.M. (2011). The importance of Innovation and its Crucial Role in Growth, Survival and Success of Organizations. Procedia Technology, 1 (2012) $535-538$.

Turriago, A. (2014). Innovación y Cambio Tecnológico en la Sociedad del Conocimiento. 2da Edicion. Bogotá: Ecoe Ediciones.

Vara-Horna, Arístides (2010). ¿Cómo hacer una tesis en ciencias empresariales? Manual breve para los tesistas de Administración, Negocios Internacionales, Recursos Humanos y Marketing. Facultad de Ciencias Administrativas y Recursos Humanos de la Universidad de San Martín de Porres. Lima - Perú. Segunda edición (Abreviada).

Visitacion, M. (2018). The Forrester Wave ${ }^{\mathrm{TM}}$ : Collaborative Work Management Tools For The Enterprise, Q4 2018. Forrester Research, Inc. 